\title{
Implantation d'un programme de zoothérapie en milieu hospitalier pour enfants atteints de cancer : une étude descriptive
}

\begin{abstract}
Par Johanne Gagnon, France Bouchard, Marie Landry, Marthe Belles-Isles, Martine Fortier et Lise Fillion
\end{abstract}

\section{Résumé}

Les enfants atteints d'un cancer doivent faire face à la maladie, à de fréquentes hospitalisations, à des traitements agressifs, et à plusieurs effets secondaires des traitements. La combinaison de ces stresseurs peut entraîner des effets indésirables sur les plans biopsychosociaux. Un programme de zoothérapie, "La Magie d'un rêve», a été conçu pour les enfants hospitalisés en oncologie pédiatrique afin de promouvoir leur bien-être pendant l'hospitalisation et de faciliter leur adaptation au processus thérapeutique. L'objectif principal de cette étude préliminaire est d'évaluer, de façon descriptive, l'implantation de ce programme et ce, à partir du modèle de qualité de Donabedian. Plus spécifiquement, cette étude vise à documenter le lien observé entre le fait de bénéficier du programme, la qualité des soins, et la satisfaction des parents et des infirmières participantes.

Un total de 16 parents d'enfants et de 12 infirmières a participé à l'étude d'implantation et a constitué l'échantillon. Les données ont été recueillies au moyen de deux questionnaires d'évaluation autoadministrés destinés aux parents et d'un questionnaire destiné aux infirmières. L'évaluation de la qualité du service de zoothérapie inclut des préoccupations reliées au profil des usagers du programme, au processus d'intervention de zoothérapie, à la structure organisationnelle, et aux résultats clientèles. Il semble qu'une thérapie assistée d'un chien puisse contribuer à alléger la détresse psychologique des enfants et des parents, à faciliter leur adaptation au processus thérapeutique, et à promouvoir leur bien-être pendant l'hospitalisation. Une seconde phase du projet aura pour but de vérifier l'efficacité de l'intervention de zoothérapie en visant plus particulièrement les enfants hospitalisés pour le traitement d'une tumeur solide.

Au Canada, plus de 1200 diagnostics de cancer sont portés chaque année chez les enfants de 0 à 19 ans (Institut national du cancer du Canada, 2000), faisant de cette affection la seconde cause de décès chez les 5 à 19 ans (Santé Canada, 1996). Ces enfants doivent faire face à la maladie, à de fréquentes hospitalisations, à des traitements agressifs, et à plusieurs effets secondaires des traitements (douleurs, nausées, vomissements, et perte d'appétit). La combinaison de ces stresseurs peut entraîner des effets indésirables sur les plans biopsychosociaux.

Parmi ces effets indésirables, les enfants hospitalisés peuvent notamment manifester une détresse psychologique. Pour ceux-ci, la détresse correspond souvent à des manifestations d'anxiété, d'irritabilité, ou à des symptômes dépressifs (Enskar, Carlsson, Hamrin, \& Kreuger, 1997; Sheldon, 1997; van Dongen-Melmen \& Sanders-Woudstra, 1986). Cette détresse peut contribuer à augmenter les effets secondaires des traitements et même entraver leur déroulement comme tel. De plus, la détresse des enfants hospitalisés est souvent accompagnée par des stratégies adaptatives non efficaces tels que l'évitement cognitif et comportemental pouvant contribuer à augmenter la difficulté d'adaptation de l'enfant à la maladie, aux traitements, et au milieu hospitalier. Par ailleurs, la détresse des enfants peut avoir des répercussions sur celle des membres de leur famille et du personnel soignant.

Cette détresse observée chez certains enfants hospitalisés et leur famille, ainsi que le sentiment d'impuissance des soignants les côtoyant ont favorisé l'émergence du projet pilote de zoothérapie du Centre hospitalier universitaire de Québec (CHUQ): «La Magie d'un rêve». Né d'une initiative infirmière en 1999 , ce projet a pour but de promouvoir le bien-être des enfants atteints de cancer pendant l'hospitalisation, de diminuer ainsi leur détresse émotionnelle et de faciliter leur adaptation $\mathrm{au}$ processus thérapeutique (psychologique, physique et social) en favorisant l'émergence de liens privilégiés entre l'enfant et l'animal. Le programme de zoothérapie du CHUQ permet aux enfants accompagnés d'un parent de passer une journée complète avec un chien lors de périodes d'hospitalisation et ce, dans une chambre aménagée de façon sécuritaire, chaleureuse et familiale (Landry et al., 2000). En plus de faciliter l'adaptation de l'enfant, cette initiative peut contribuer à l'amélioration de la qualité des soins notamment en offrant un service pour lequel des résultats clientèles ont déjà été observés (repos réparateur, meilleure alimentation, exercice physique, socialisation, participation à des activités récréationnelles, verbalisation des craintes et des inquiétudes, sentiment d'être moins anxieux, et plus heureux, etc.).

La zoothérapie est définie comme étant une méthode clinique cherchant à favoriser les liens naturels et bienfaisants, qui existent entre les humains et les animaux, à des fins préventives et thérapeutiques (Daoust, 1987). Le rationnel qui sous-tend cette pratique est que l'animal stimule naturellement une réponse d'attraction et d'attachement chez l'être humain (Brodie \& Biley, 1999), se répercutant ainsi sur le bien-être de la personne. Comme le bien-être est incompatible avec l'état de détresse émotionnelle, la zoothérapie peut constituer une intervention bénéfique pour le soulagement de la détresse de l'enfant, de sa famille et des soignants.

\section{L'état des connaissances}

L'effet de la zoothérapie sur l'allégement de la détresse n'a été que très peu documenté empiriquement. Les rares études portant sur le sujet ont recours à des devis descriptifs qui suggèrent que la zoothérapie puisse avoir des effets bénéfiques (pour une recension, voir Brodie et Biley, 1999; Poleshuck, 1997). La majorité de ces études descriptives a été réalisée auprès de clientèles très différentes (personnes âgées ou présentant des problèmes de santé mentale) de celle retenue ici (enfants atteints de cancer) ou utilisant des modalités de zoothérapie non transférables au milieu hospitalier. Considérant de plus leur faible validité interne, les résultats de ces travaux ont été exclus de cet article. Quelques études documentent la zoothérapie en milieu hospitalier et d'autres s'intéressent à l'application de celle-ci auprès de populations d'enfants. Ces études sont brièvement résumées dans les sections suivantes.

Certaines études documentent l'implantation de programmes de zoothérapie en milieu de soins. Par exemple, la satisfaction d'une

Johanne Gagnon, inf., PhD, est membre de la Faculté des sciences infirmières de l'Université Laval, Québec. France Bouchard, B.Sc.inf., inf., et Marie Landry, inf., oeuvrent à l'Unité pédiatrie du CHUL à Québec. Marthe Belles-Isles, PhD, oeuvre au Département de Biochimie clinique au CHUL. Martine Fortier, B.Sc., est à l'École de psychologie de l'Université Laval, et Lise Fillion, PhD, est membre de la Faculté des sciences infirmi “eres de l'Université Laval et du Centre de recherche en cancérologie de l'Université Laval, CHUQ-HDQ. 
clientèle adulte face à l'implantation d'un tel programme dans une unité de soins cardiaques fut évaluée à l'aide de cinq questions (Cole \& Gawlinski, 1995). Le programme impliquait 120 visites d'un chien et son maître. Les patients se disent satisfaits, mentionnant que les visites les rendent plus joyeux, calmes, et moins seuls. Par ailleurs, les résultats d'une étude quasi-expérimentale effectuée auprès de 36 résidents d'un centre médical, démontrent une association entre la présence d'un chien et une augmentation des interactions sociales (Fick, 1992). Une relation similaire a été observée lors d'une étude expérimentale auprès de 53 résidents d'un centre d'hébergement (Perelle \& Granville, 1998). Dans cette dernière étude, notons toutefois, que l'amélioration observée au niveau des interactions sociales lors de la zoothérapie ne se maintient pas dans le temps. De façon générale, et ce malgré les limites méthodologiques associées aux devis corrélationnels retenus, ces trois études soutiennent la faisabilité de l'implantation d'un programme de zoothérapie dans un milieu médical et décrivent des effets bénéfiques au niveau de l'état émotionnel et des comportements sociaux susceptibles de favoriser l'adaptation des clientèles adultes au milieu hospitalier.

Quelques études sur la zoothérapie auprès des enfants ont été recensées. Parmi elles, figure une étude expérimentale réalisée auprès de 12 enfants autistiques fréquentant un centre spécialisé (Redefer \& Goodman, 1989). Les participants ont bénéficié de 18 rencontres de 20 minutes avec un chien et le thérapeute habituel. Les résultats démontrent une baisse des comportements autistiques chez les participants de même qu'une augmentation des comportements sociaux appropriés. Une autre étude, celle-ci exploratoire, a été réalisée auprès d'un échantillon de 12 enfants éprouvant des problèmes de comportements et des difficultés scolaires (Mallon, 1994b). Les données qualitatives et quantitatives obtenues suggèrent que la présence d'un chien pourrait être associée à des effets bénéfiques sur les plans émotionnel, physique, et social. De plus, les auteurs estiment que les bénéfices de l'intervention effectuée pourraient surpasser les coûts. Par ailleurs, deux études de cas auprès de garçons ayant une perturbation émotive décrivent la zoothérapie comme offrant une opportunité aux participants de se sentir davantage en contrôle et d'exprimer un sentiment de fierté et d'accomplissement (Kogan, Granger, Fitchett, Helmer, \& Young, 1999). Les résultats de ces trois études suggèrent que la zoothérapie auprès des enfants pourrait avoir un effet positif sur l'état émotionnel et pourrait augmenter les stratégies comportementales efficaces contribuant ainsi à faciliter l'adaptation de l'enfant.

La recension a permis de repérer deux seules études où la zoothérapie était à la fois appliquée en milieu hospitalier et destinée à une jeune clientèle. Une première étude qualitative décrit la réponse d'adolescents hospitalisés à la présence d'un chien sur l'unité psychiatrique (Bardill \& Hutchinson, 1997). Une approche ethnographique est utilisée, permettant de comprendre le point de vue des participants face à cette expérience. Le chien est décrit par les adolescents à la fois comme une composante du milieu, comme un ami, et comme un thérapeute. L'animal est aussi décrit comme un catalyseur au niveau des interactions sociales et se voit attribuer des qualités humaines telles que réconfortante, amicale, et attentive. Le chien est accueilli favorablement par les adolescents et par l'unité psychiatrique. La seconde étude documente l'implantation d'un programme de zoothérapie canine dans un hôpital pour enfants en collaboration avec des étudiants en médecine vétérinaire (Teeter, 1997). L'objectif du programme est de minimiser la douleur émotionnelle associée à l'hospitalisation chez l'enfant et sa famille en leur permettant de se rassembler dans un lieu calme pour être en compagnie de deux ou trois chiens, pendant une période d'environ une heure. L'auteur y annonce l'évaluation prochaine de l'efficacité et de l'efficience du programme.

L'ensemble des résultats provenant d'études descriptives et corrélationnelles soutient la présence d'une relation positive entre la zoothérapie et l'amélioration de l'état émotionnel. L'interprétation de ces données appuie la pertinence d'implanter un programme de zoothérapie en oncologie afin de faciliter l'adaptation de l'enfant à sa maladie, aux traitements, et à son hospitalisation. Par exemple, chez l'enfant malade, la zoothérapie pourrait diminuer l'anxiété et les symptômes dépressifs, briser l'isolement, et augmenter les interactions sociales. Elle pourrait aussi augmenter la collaboration de l'enfant aux traitements et contribuer à diminuer l'inconfort physique associé aux procédures douloureuses (Barba, 1995; Brodie \& Biley, 1999; Poleshuck, 1997; Struck \& Brady, 1998). Dans un contexte hospitalier, l'animal pourrait être d'un grand support par sa capacité d'offrir une compagnie agréable et affectueuse, de même que par le sentiment d'être écouté qu'il procure (Mallon, 1994a). De plus, il aiderait à préserver le caractère normal d'une situation (Brodie \& Biley). L'animal, de par sa chaleur et sa fourrure, pourrait aussi jouer un rôle sécurisant et réconfortant (Jonas \& Feline, 1981; Mallon, 1994b).

Selon la théorie transactionnelle de stress suggérée initialement par Lazarus et Folkman (1984), plusieurs mécanismes contribueraient à diminuer la détresse de l'enfant et à faciliter son adaptation au stresseur complexe que constitue le cancer, ses traitements, et les nombreuses hospitalisations. En contribuant à faciliter l'adaptation de l'enfant à ce stresseur complexe, l'implantation d'un programme de zoothérapie pourrait également faciliter la compliance aux traitements, diminuer les symptômes, et les effets secondaires des traitements et finalement, se répercuter sur la qualité des soins infirmiers. C'est pourquoi, cette présente étude a pour but d'évaluer, à l'aide du modèle de Donabedian (1980), la qualité du service de zoothérapie offert, par les infirmières, à la clientèle d'oncologie pédiatrique du CHUQ.

Figure 1 - Modèle pour l'évaluation de résultats (Morin, 1998) inspiré de Donabedian (1980)

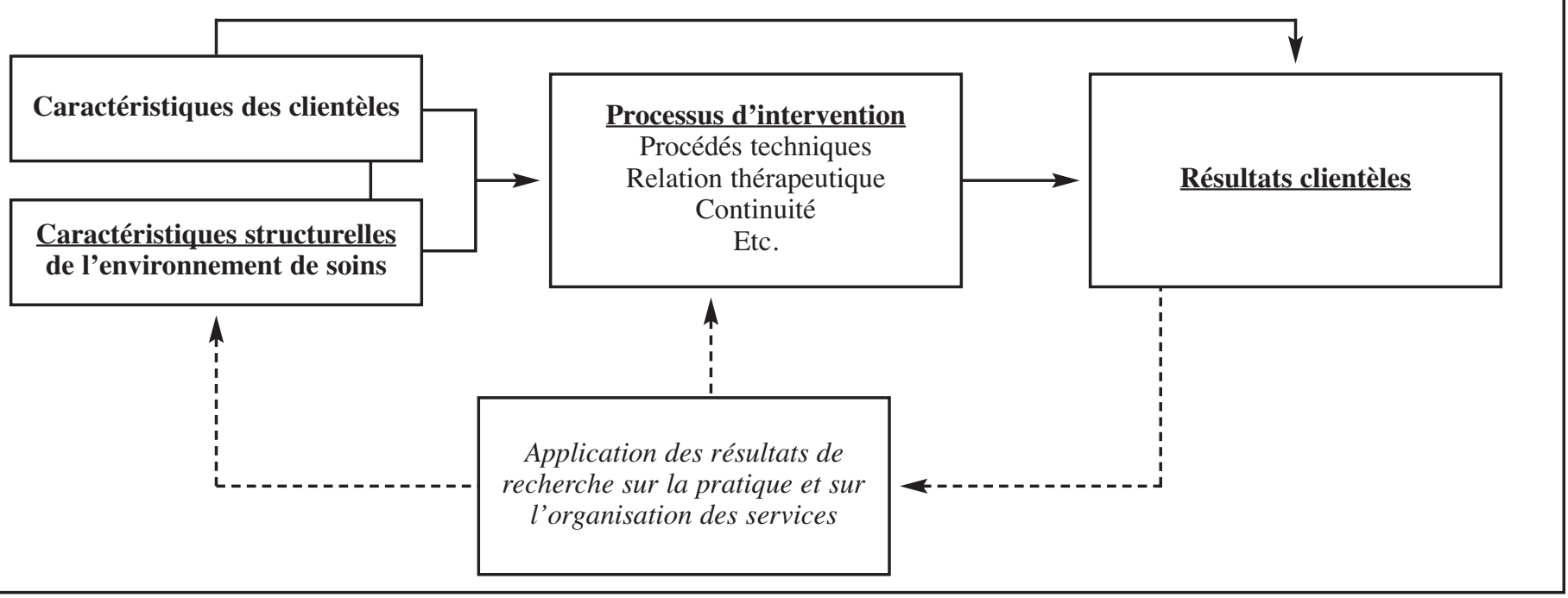




\section{Le cadre d'analyse}

Le modèle de Donabedian est souvent retenu pour l'évaluation de la qualité (Brunelle, 1993; Haddad, Roberge, \& Pineault, 1997). Ce modèle propose une approche trilogique où l'évaluation de la qualité peut être effectuée selon la structure, le processus ou les résultats.

Les approches pour mesurer la qualité des soins et des services ont porté traditionnellement sur des éléments de la structure permettant ainsi d'examiner les aspects organisationnels et physiques ainsi que les caractéristiques spécifiques de l'établissement (Donabedian, 1980; Zimmer, 1980). Dans le domaine des soins infirmiers, l'évaluation de la qualité a particulièrement ciblé les processus de soins comme champs d'intérêt. Le processus englobe toutes les activités qui se déroulent entre les dispensateurs et les patients (Laurin, 1988). Plus récemment, la contribution des soins infirmiers à des objectifs de recouvrement, de maintien, d'amélioration de la santé, ou leur contribution à une mort sereine est analysée dans la perspective des résultats (Morin, 1997). L'évaluation des résultats inclut à la fois les «Output» qui font référence aux résultats plus près de la production de services, et les «Outcome», qui font référence aux changements de l'état de santé (Saucier \& Brunelle, 1995).

Dans cette perspective "résultats", nous retenons le modèle de Morin (1998) pour l'évaluation du projet pilote de zoothérapie. Selon cette auteure, évaluer les résultats est établir «le lien entre des éléments des structures de soins et des bénéfices objectifs ou subjectifs pour les patients, tout en mettant simultanément en lumière certains aspects des processus de soins qui les ont vraisemblablement induits» (Morin, 1998, p. 25). Ce modèle, fortement inspiré de la trilogie de Donabedian (1980), schématise (figure 1) les liens qui peuvent être établis entre les éléments de la structure organisationnelle, du processus d'intervention, et des résultats tels que vécus par les clientèles visées soit les patients et leur famille.

\section{L'objectif principal de l'étude}

A partir du cadre de Donabedian (1980) et du modèle de Morin (1998) pour l'évaluation de résultats, le présent article porte sur l'étude descriptive de l'implantation d'un programme de zoothérapie en milieu hospitalier. Elle est la première, selon notre recension, à s'intéresser spécifiquement à une clientèle en oncologie pédiatrique et à permettre à l'enfant de passer une journée entière en compagnie d'un chien. L'objectif principal de cette étude est d'évaluer l'implantation du programme de zoothérapie «La Magie d'un rêve» en décrivant le lien observé entre le fait de bénéficier du programme, la qualité des soins, et la satisfaction des parents et des infirmières participantes.

\section{La méthode}

\section{Devis et variables de l'étude}

Il s'agit d'une étude évaluative de l'implantation d'un programme de zoothérapie en milieu hospitalier utilisant un devis descriptif. Le programme de zoothérapie constitue la première variable d'intérêt. Il a été développé pour la clientèle d'oncologie pédiatrique du CHUQ afin de promouvoir le bien-être des enfants atteints de cancer pendant leur hospitalisation. Des éléments de satisfaction et de qualité des soins dont entre autres, l'expression de sentiments; la fidélité aux traitements; l'autonomie fonctionnelle; le repos, l'alimentation, et l'exercice physique; la participation à des activités récréationnelles constituent les variables associées.

\section{Population et échantillon}

La population à l'étude était constituée, d'une part de parents d'enfants atteints de cancer hospitalisés au CHUQ et d'autre part, d'infirmières travaillant auprès de cette clientèle. Le CHUQ comprend 12 lits d'oncologie pédiatrique et dispense des soins tertiaires à une population d'environ 35 à 45 nouveaux cas de cancer infantile par année. Par ailleurs, le traitement actif en oncologie pédiatrique peut s'échelonner sur plusieurs mois (plus de 2 ans pour la leucémie) ainsi, la cohorte d'enfants traités simultanément représente un volume beaucoup plus élevé.

Les enfants participants à l'étude devaient avoir reçu un diagnostic de cancer et être âgés d'au moins deux ans. Le parent de l'enfant devait être capable de parler, lire et écrire en français, et devait fournir son consentement écrit. L'infirmière participant à l'étude devait prendre soin d'un enfant bénéficiant de la zoothérapie et devait aussi fournir son consentement écrit. Les critères d'exclusion étaient un test cutané positif aux allergènes canins, une neutropénie sévère (moins de 500 neutros $/ \mu \mathrm{L}$ de sang), une chirurgie récente, ou être sous technique stérile et un comportement agressif, un trouble développemental, ou un déficit cognitif sévère de l'enfant empêchant une interaction normale avec un animal. Un total de 16 parents d'enfants et de 12 infirmières a participé à l'étude d'implantation et a constitué l'échantillon.

\section{Déroulement de l'étude}

L'idée du projet a d'abord vu le jour en juin 1998 pour débuter officiellement à l'automne 1999 et ce, sous la forme d'un projet pilote pour une période de 12 mois (pour plus d'information concernant le projet pilote, voir l'article de Bouchard, Landry, Belles-Isles, \& Gagnon, 2004). C'est ainsi qu'à l'été 1999, une chambre privée dédiée uniquement à la zoothérapie a été aménagée à l'unité d'hémato-oncologie pédiatrique du CHUQ et, en octobre 1999, un premier enfant bénéficiait du service de zoothérapie. L'étude d'implantation du programme de zoothérapie a été effectuée au cours des six premiers mois du projet pilote, soit d'octobre 1999 à mars 2000. Un total de 39 services sur 53 services enregistrés a été évalué. Certains n'ont pas été évalués puisqu'il a fallu un certain temps d'ajustement avant de débuter correctement l'évaluation.

Lors d'une séance de zoothérapie, l'enfant passe la journée complète, soit de 8 heures à 16 heures, en compagnie d'un chien. Le chien en question doit auparavant subir un examen physique et comportemental rigoureux. Les séances se déroulent dans une chambre spécialement aménagée à cette fin. L'enfant y reçoit des soins infirmiers et médicaux tout en ayant la possibilité d'interagir et de jouer avec le chien. Un des parents, ou encore une personne responsable de l'enfant, doit être présente tout le long de la journée. Cette personne encourage l'interaction entre l'enfant et l'animal.

Au cours de l'étude, la confidentialité a été assurée par recours à des ententes de non-divulgation. Aucune information nominative n'est apparue sur les outils de collecte des données. De plus, lors du compte rendu écrit des données et lors de présentations orales, les responsables de l'étude se sont engagés à ne pas utiliser d'information permettant de reconnaître un sujet particulier. Les responsables de l'étude ont également respecté les règles d'éthique propres à l'établissement de santé. Il a été convenu que les parents seraient identifiés et contactés par les infirmières afin de connaître leur intérêt de participer à l'étude. Une fois leur consentement obtenu, le questionnaire leur serait distribué par l'infirmière responsable des soins de leur enfant qui a elle-même consenti à participer à l'étude et à remplir un questionnaire destiné au personnel infirmier.

\section{Instruments et collecte des données}

Les données ont été recueillies au moyen de trois questionnaires d'évaluation auto-administrés (deux questionnaires destinés aux parents et un questionnaire destiné aux infirmières). Les questionnaires ont été élaborés à partir des écrits (Cole \& Gawlinski, 1995; Fick, 1992; Hawley, 1998; Jorgenson, 1997; Martin, 1993; Perelle \& Granville, 1998; Ruckert, 1994; Saylor, 1998). La validité de contenu a été examinée par un panel d'experts constitué de trois infirmières ayant une expertise en oncologie pédiatrique, une connaissance pratique de la zoothérapie, et d'un professeur en mesure et évaluation de l'Université Laval. Un pré-test a également été réalisé auprès de trois mères dont l'enfant avait bénéficié du service de zoothérapie. Puisque nous étions dans un processus d'implantation, les modalités entourant les visites du chien ont évolué au gré de certains correctifs apportés suite aux 
évaluations et commentaires des parents et du personnel. Ainsi, les questionnaires ont subi de fréquentes transformations mineures tout au long de l'évaluation de l'implantation du programme.

La littérature mentionne que selon l'approche privilégiée (perspective des patients/familles ou perspective des professionnels de la santé), les perceptions de la qualité des soins, et des services ont tendance à différer (Brunelle, 1993; Haddad et al., 1997; LudwigBeymer et al., 1993; Meisenheimer, 1991; St-Hilaire \& Erickson, 1999). Ainsi, lors de l'évaluation de la qualité d'une nouvelle approche, telle la zoothérapie, il était important de tenir compte à la fois des opinions des patients/familles ainsi que de celles des intervenants; raison pour laquelle les questionnaires visaient conjointement les perceptions des parents et des infirmières relatives à la qualité du service de zoothérapie.

Un premier questionnaire a été administré à tous les parents dont l'enfant a bénéficié du service de zoothérapie et à toutes les infirmières qui prenaient soin de ces enfants entre les mois d'octobre 1999 et mars 2000. Ensuite, toujours pour cette même période, un deuxième questionnaire a été administré aux parents dont l'enfant a bénéficié du service de zoothérapie plus de 3 fois et même plus de 5 fois au cours de leurs nombreuses hospitalisations. Certaines données socio-démographiques et médicales ont aussi été colligées afin de décrire les caractéristiques des enfants et de leurs parents. Des analyses descriptives (quantitatives et qualitatives) ont été réalisées sur l'ensemble des données recueillies.

Le premier questionnaire, destiné aux parents, est composé de trois parties. La première partie comprend 20 énoncés regroupés en trois sous-échelles (voir tableau 1). Ces énoncés se mesuraient sur une échelle de type Likert à 5 points, variant entre 0 , «tout à fait en désaccord» et 4 , «tout à fait d'accord». La deuxième partie a trait à la satisfaction des parents se mesurant aussi sur une échelle de 0 , «pas du tout satisfait» à 4 , «très satisfait». Enfin, la dernière partie du premier questionnaire se compose de trois questions ouvertes qui ont fait l'objet d'analyses de contenu qualitatives. Le deuxième questionnaire destiné aux parents, comprend deux parties très similaires aux deux premières parties du premier questionnaire. Seule l'échelle de mesure diffère dont les points varient entre 0 , «pas de différence » et 4 , « différence capitale».

Le questionnaire destiné au personnel infirmier est constitué de quatre parties. Les deux premières sont semblables à celles du premier questionnaire destiné aux parents. La troisième partie est composée de 12 énoncés se rapportant à l'organisation du travail relative à la zoothérapie. Finalement, la dernière partie comporte deux questions ouvertes qui ont, à nouveau, fait l'objet d'analyses de contenu qualitatives.

\section{Les résultats}

L'analyse des résultats des trois questionnaires d'évaluation de l'implantation du projet a permis de répondre à notre objectif principal et ainsi, d'évaluer, de façon descriptive, certains effets de la zoothérapie grâce aux réponses des parents et des soignants.

\section{Profil des répondants}

L'analyse des données socio-démographiques et médicales a démontré que parmi les 16 enfants ayant bénéficié de la zoothérapie, $89 \%$ étaient âgés entre 3 et 13 ans et $54 \%$ bénéficiaient du service pour la première fois. Puis, $46 \%$ des enfants étaient de sexe masculin et $54 \%$ de sexe féminin. La maladie de l'enfant avait été diagnostiquée depuis moins d'un an chez $77 \%$ des enfants dont $29 \%$ étaient atteints d'une leucémie. Les 12 infirmières possédaient entre 2 et 20 années d'expérience et la majorité travaillait de jour, à temps complet, à l'unité d'oncologie pédiatrique.

\section{Éléments de la qualité des soins (perspectives des parents et des infirmières)}

Les principaux résultats ont été regroupés selon les quatre éléments du modèle de Morin, tel qu'illustré à la figure 1, soit: 1) les caractéristiques des clientèles, 2) les caractéristiques structurelles de l'environnement de soins, 3) le processus d'intervention, et 4) les résultats clientèles. Le tableau 1 présente les pourcentages d'accord et d'indécis des parents et des infirmières relativement à certains résultats attendus chez la clientèle pédiatrique et retrouvés dans les

Tableau 1: Sommaire des résultats clientèles: Pourcentages d'accord et d'indécis des parents et des infirmières

\begin{tabular}{|c|c|c|c|c|}
\hline \multirow{2}{*}{$\begin{array}{l}\text { Résultats clientèles } \\
\text { L'enfant }\end{array}$} & Parents & Inf. & Parents & Inf. \\
\hline & \multicolumn{2}{|c|}{$\%$ accord } & \multicolumn{2}{|c|}{$\%$ indécis } \\
\hline $\begin{array}{l}\text { Avoir la responsabilité du chien a permis à mon enfant de: } \\
\text { Développer un sentiment «d'être essentiel à quelqu'un» } \\
\text { Exprimer un sentiment de fierté, d'accomplissement } \\
\text { Mieux accepter l'hospitalisation } \\
\text { Être plus réceptif et fidèle aux traitements } \\
\text { Soulager ou diminuer son anxiété }\end{array}$ & $\begin{array}{c}100 \\
96 \\
92 \\
88 \\
79\end{array}$ & $\begin{array}{l}100 \\
100 \\
100 \\
100 \\
100\end{array}$ & $\begin{array}{c}0 \\
4 \\
4 \\
8 \\
17\end{array}$ & $\begin{array}{l}0 \\
0 \\
0 \\
0 \\
0\end{array}$ \\
\hline $\begin{array}{l}\text { En présence du chien,j'ai eu l'impression que mon enfant: } \\
\text { Avait développé un lien d'amitié avec l'animal } \\
\text { Semblait plus heureux (plus souriant, plus ricaneux) } \\
\text { Avait plus confiance en lui } \\
\text { Se sentait plus «normal» ou «moins malade» } \\
\text { Était plus motivé à poursuivre son hospitalisation } \\
\text { Était plus autonome dans la satisfaction de ses besoins }\end{array}$ & $\begin{array}{l}96 \\
92 \\
78 \\
74 \\
74 \\
50\end{array}$ & $\begin{array}{c}100 \\
100 \\
100 \\
95 \\
100 \\
95\end{array}$ & $\begin{array}{l}4 \\
4 \\
18 \\
22 \\
22 \\
32\end{array}$ & $\begin{array}{l}0 \\
0 \\
0 \\
5 \\
0 \\
5\end{array}$ \\
\hline $\begin{array}{l}\text { Le simple fait d'être en présence du chien a encouragé mon enfant à: } \\
\text { Sortir plus souvent de sa chambre (interactions) } \\
\text { Participer à des activités récréationnelles (jeux, dessins) } \\
\text { Socialiser et partager avec les autres enfants ou adultes } \\
\text { Se reposer } \\
\text { Surmonter certaines difficultés } \\
\text { Se rapprocher davantage des autres } \\
\text { Mieux s'alimenter } \\
\text { Faire de l'exercice physique } \\
\text { Verbaliser ses craintes et inquiétudes }\end{array}$ & $\begin{array}{l}74 \\
70 \\
65 \\
63 \\
60 \\
55 \\
48 \\
40 \\
40\end{array}$ & $\begin{array}{l}35 \\
57 \\
58 \\
67 \\
96 \\
75 \\
67 \\
66 \\
48\end{array}$ & $\begin{array}{l}22 \\
25 \\
25 \\
23 \\
40 \\
36 \\
29 \\
35 \\
50\end{array}$ & $\begin{array}{c}59 \\
20 \\
37 \\
29 \\
4 \\
20 \\
24 \\
24 \\
52\end{array}$ \\
\hline
\end{tabular}


écrits. Nous pouvons remarquer que des différences émergent entre les opinions des parents et celles des infirmières. Les infirmières sont, de façon générale, plus souvent en accord avec les affirmations que les parents. En effet, les infirmières ont déclaré être moins d'accord que les parents à trois seules affirmations sur 20 soit: le simple fait d'être en présence du chien a encouragé l'enfant 1) à sortir plus souvent de sa chambre, 2) à participer à des activités récréationnelles, ainsi 3) qu'à socialiser et partager avec les autres.

Le tableau 2 présente quelques pourcentages de satisfaction des parents et des infirmières en regard du niveau de satisfaction générale; de l'importance et de la structure d'un tel programme; des attentes des parents; et de l'organisation du travail.

D'autres éléments de satisfaction ont aussi été colligés suite à l'analyse de contenu des questions ouvertes. Ces éléments se rapportent à la structure organisationnelle qui est définie comme confortable, calme, relaxante, paisible, douce, et chaleureuse. Puis, certains autres éléments se rapportent au processus d'intervention qui utilise entre autres, des chiens attachants, chaleureux, bons, et libres. Enfin, des éléments se rapportent plus spécifiquement aux résultats clientèles soit 1) l'enfant atteint de cancer, qui est plus autonome, qui s'alimente mieux et qui dit adorer l'hôpital, 2) ses parents, qui affirment que l'hospitalisation est moins triste, que c'est une journée de bonheur et que cela aide au moral, et 3) le personnel infirmier, qui se dit plus gai et enjoué, plus motivé, plus positif et que le programme facilite le travail et remonte le moral.

Un deuxième questionnaire a également été administré aux sept parents dont l'enfant avait bénéficié du service de zoothérapie plus de 3 fois et même plus de 5 fois au cours de ses nombreuses hospitalisations. Depuis le début des séances de zoothérapie, la moitié des parents affirment avoir remarqué une différence majeure chez leur enfant en ce qui concerne a) l'amélioration du bien-être général de l'enfant, b) le développement d'un lien d'amitié avec l'animal, et c) la participation de l'enfant à des activités récréationnelles. Plus de la moitié des parents affirment avoir également remarqué chez leur enfant une amélioration modérée en ce qui concerne la fidélité aux traitements et la motivation à poursuivre l'hospitalisation, ainsi qu'une différence majeure en ce qui concerne la distraction à l'hôpital. Enfin, la moitié des parents affirment avoir remarqué une différence majeure au cours de cette journée en ce qui concerne la peur, la douleur et l'inconfort de leur enfant liés aux traitements et aux interventions.

Tableau 2: Sommaire des résultats clientèles: Pourcentages de satisfaction des parents et des infirmières

\begin{tabular}{|c|c|}
\hline Résultats clientèles & $\begin{array}{c}\text { Parents Infirmières } \\
\% \text { accord }\end{array}$ \\
\hline $\begin{array}{l}\text { Très satisfait du programme } \\
\text { Satisfait du programme } \\
\text { Très important pour l'enfant malade } \\
\text { Important pour l'enfant malade } \\
\text { Attentes dépassées } \\
\text { Attentes entièrement rencontrées } \\
\text { Attentes partiellement rencontrées } \\
\text { Recommandation à d'autres } \\
\text { Influence le choix de l'hôpital ou de l'unité }\end{array}$ & $\begin{array}{c}96 \\
4 \\
65 \\
35 \\
32 \\
52 \\
17 \\
100 \\
92\end{array}$ \\
\hline $\begin{array}{l}\text { Programme bien structuré } \\
\text { Ajustements corrects à chaque visite } \\
\text { Impact positif sur le travail }\end{array}$ & $\begin{array}{c}\text { \% accord } \\
94 \\
94 \\
95\end{array}$ \\
\hline $\begin{array}{l}\text { Apporte un surplus de travail } \\
\text { Nuit au travail sur l'unité } \\
\text { Prend trop de place sur l'unité }\end{array}$ & $\begin{array}{c}\text { \% désaccord } \\
100 \\
100 \\
100\end{array}$ \\
\hline
\end{tabular}

Intégration des principaux résultats au modèle d'évaluation

La figure 2 intègre les principaux résultats de cette étude d'implantation au modèle d'évaluation de Morin (1998).

Ainsi, nous pouvons remarquer sur cette figure que l'évaluation de la qualité du service de zoothérapie inclut des préoccupations reliées 1) au profil des usagers du programme soit l'âge, le sexe, le type de cancer, et le moment du diagnostic, 2) au processus d'intervention de zoothérapie soit la relation avec le chien et l'utilisation de celle-ci par les infirmières comme moyen thérapeutique, 3) à la structure organisationnelle soit la chambre, la journée complète, le protocole rigoureux, les différents outils papiers et audio-visuels, les activités reliées et l'accessibilité du service, et enfin 4) aux résultats clientèles soit l'enfant, le parent et l'infirmière.

\section{La discussion}

L'objectif principal de cette étude était d'évaluer, de façon descriptive, l'implantation du programme de zoothérapie à partir du cadre de Donabedian (1980) et, plus spécifiquement du modèle de Morin (1998). Plus précisément, cette étude voulait documenter le lien observé entre le fait de bénéficier du programme, la qualité des soins et la satisfaction des parents et des infirmières participantes. Étant donné le devis descriptif utilisé, il est possible que les résultats soient attribuables à l'ensemble du processus zoothérapeutique (chambre et ambiance, présence du chien, contact différent avec l'infirmière, et activités reliées) plutôt qu'à l'effet isolé de la seule présence du chien.

\section{Rôle bénéfique de la zoothérapie}

La présente étude a permis de mettre en évidence certaines dimensions pour lesquelles une amélioration a été observée. Ainsi, les résultats soulèvent le rôle potentiellement bénéfique de la zoothérapie sur les dimensions physiques (repos, alimentation, exercice), sociales (socialiser, se rapprocher des autres, sortir plus souvent de sa chambre, participer à des activités récréationnelles), affectives (diminution de l'anxiété, semble plus heureux, verbalise ses craintes et inquiétudes), ainsi que sur l'adaptation (mieux accepter l'hospitalisation, surmonte certaines difficultés, être plus réceptif au traitement, plus autonome, plus motivé à poursuivre l'hospitalisation), et l'estime de soi (sentiments de fierté, d'accomplissement, d'être plus normal, d'être essentiel à quelqu'un, d'avoir plus confiance en soi). Ces résultats, de même que le niveau de satisfaction obtenu par les différents acteurs, convergent étroitement avec ceux retrouvés dans la littérature (Barba, 1995; Bardill \& Hutchinson, 1997; Brodie \& Biley, 1999; Jonas \& Feline, 1981; Kogan et al., 1999; Mallon, 1994b; Poleshuck, 1997; Struck \& Brady, 1998; Teeter, 1997). De plus, ces données préliminaires ont supporté l'élaboration de la deuxième phase de la recherche, sur l'efficacité du programme, qui a débuté en janvier 2003.

\section{Limites de l'étude}

Les limites de l'étude sont celles inhérentes à une étude descriptive d'évaluation de l'implantation d'un projet pilote. Parmi celles-ci, notons entre autres, la petite taille de l'échantillon et sa non-représentativité ainsi que l'absence de qualités psychométriques des questionnaires (validité et fiabilité) qui limitent la validité des données. De plus, la nature des énoncés du questionnaire peut avoir eu un effet suggestif sur les résultats attendus et avoir influencé les réponses des parents et du personnel infirmier. Il est aussi important de noter que le pourcentage d'indécis présenté au tableau 1 est parfois éloquent (allant jusqu'à 52\%). Cette constatation est peut-être due à la nouveauté de cette thérapie et à la difficulté, pour les participants, de se prononcer. Cependant, le fait de cerner un grand nombre de bénéfices potentiels et de décrire un programme de zoothérapie unique et innovateur peut être considéré comme des forces importantes de cette étude, qui se veut une première étape de l'évaluation. 


\section{Implications pour la pratique ef la recherche}

L'infirmière occupe une place privilégiée pour assurer le bienêtre de l'enfant hospitalisé pour un cancer et de sa famille. Il semble qu'une thérapie assistée d'un chien puisse contribuer à alléger la détresse psychologique des enfants et des parents, à faciliter leur adaptation au processus thérapeutique, et à promouvoir leur bien-être pendant l'hospitalisation. D'autres études sont cependant nécessaires afin de mieux comprendre le processus d'action de cette nouvelle thérapie et de supporter empiriquement son utilisation. C'est pourquoi, une deuxième phase d'évaluation de l'efficacité du programme de zoothérapie a été élaborée.

En effet, le projet pilote étant maintenant terminé, le programme a été reconnu officiellement à l'automne 2000 par le conseil d'administration du CHUQ et un budget de fonctionnement a été alloué pour la continuité formelle de celui-ci. Le développement et l'implantation du programme de zoothérapie constituaient la première phase du projet. Plusieurs effets positifs ont été observés au cours de l'étude d'implantation. Toutefois, l'efficacité du programme de zoothérapie demeure à documenter. La seconde phase du projet, présentement en cours, a pour but de vérifier l'efficacité de l'intervention de zoothérapie en visant plus particulièrement les

Figure 2 - Intégration des résultats de l'étude au modèle de Morin (1998) inspiré de Donabedian (1980).

Modèle pour l'évaluation de résultats

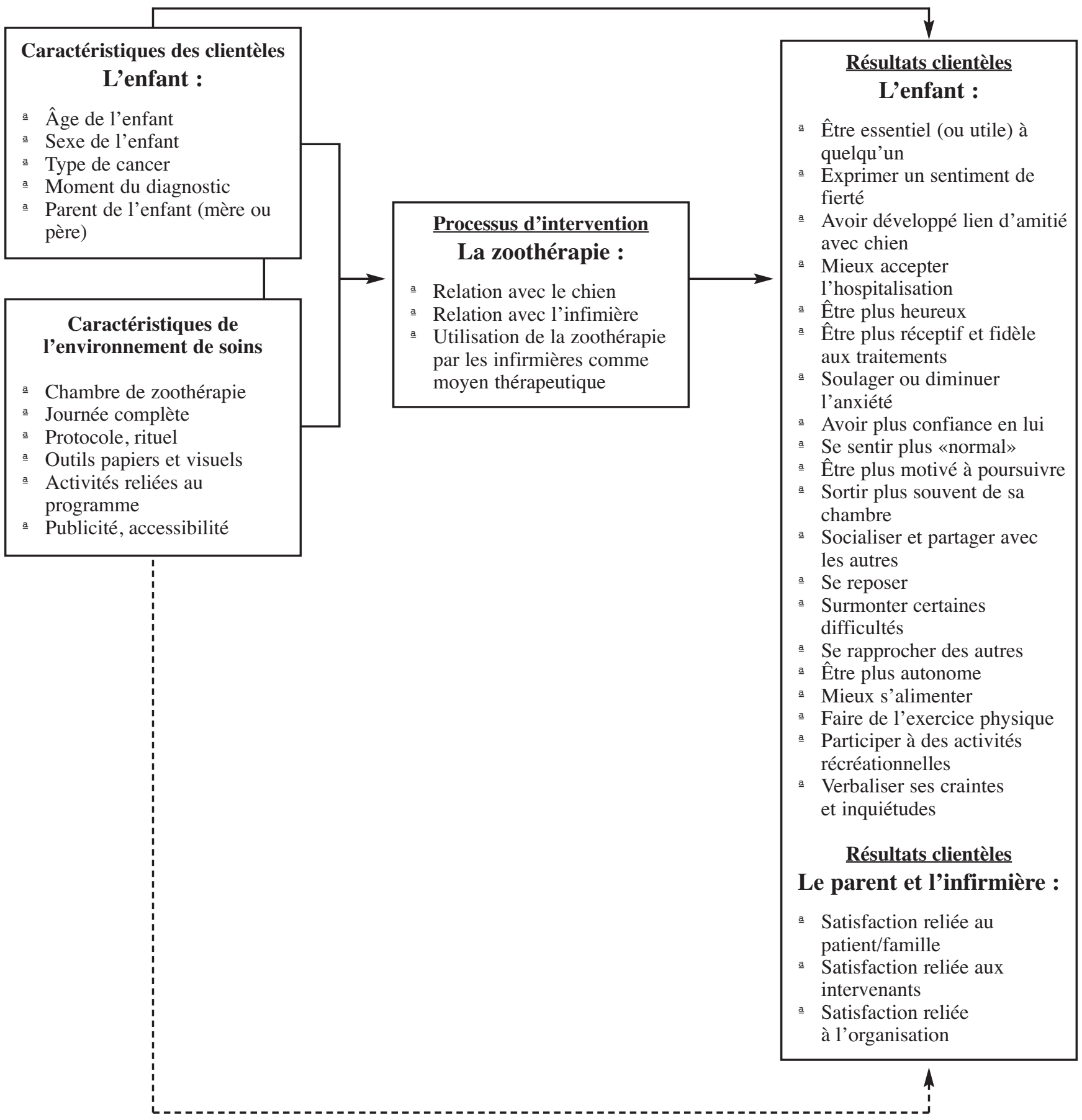


enfants hospitalisés pour le traitement d'une tumeur solide. Cette clientèle a été choisie parce qu'elle reçoit généralement de la chimiothérapie sur une longue période de temps, ce qui est susceptible d'être associé à une détresse psychologique marquée. Plus spécifiquement, les objectifs de recherche sont de: a) vérifier si la zoothérapie est associée i) à une amélioration significative de l'humeur (e.g., anxiété, dépression), et d'autres indices de fonctionnement (e.g., interactions sociales, niveau d'activité), b) à une diminution des indices physiologiques de stress (e.g., cortisol, tension artérielle), de même qu'à une diminution des effets secondaires liés à la chimiothérapie (e.g., nausées, douleur, pertes d'appétit, troubles du sommeil) tels qu'évalués par les parents et les enfants eux-mêmes; et c) vérifier si la zoothérapie est associée à une meilleure adaptation des parents vis-à-vis de la maladie de leur enfant.

\section{Références}

Barba, B.E. (1995). The positive influence of animals: Animal-assisted therapy in acute care. Clinical Nurse Specialist, 9(4), 199-202.

Bardill, N., \& Hutchinson, S. (1997). Animal-assisted therapy with hospitalized adolescents. Journal of Child and Adolescent Psychiatric Nursing, 10(1), 17-24.

Bouchard, F., Landry, M., Belles-Isles, M., \& Gagnon, J. (2004). La zoothérapie en oncologie pédiatrique "La Magie d'un rêve": Une expérience pilote. Revue canadienne de soins infirmiers en oncologie, 14(1), 10-13.

Brodie, S.J., \& Biley, F.C. (1999). An exploration of the potential benefits of pet-facilitated therapy. Journal of Clinical Nursing, 8, 329-337.

Brunelle, Y. (1993). La qualité des soins et services: Un cadre conceptuel. Ministère de la Santé et des Services Sociaux, Direction générale de la planification et de l'évaluation. Collection Études et Analyses, 16, Québec.

Cole, M.K., \& Gawlinski, A. (1995). Animal-assisted therapy in the Intensive Care Unit. Research Utilization, 30(3), 529-537.

Daoust, S. (1987). Zoothérapie. L'Institut canadien de zoothérapie en collaboration avec la Fondation Gannett (Médiacom) Lafer Inc.

Donabedian, A. (1980). Explorations in quality assessment and monitoring - Volume I - The definition of quality and approaches to its assessment. Ann Arbor: University of Michigan.

Enskar, K., Carlsson, M., Hamrin, E., \& Kreuger, A. (1997). Life situation and problems as reported by children with cancer and their parents. Journal of Pediatric Oncology Nursing, 14(1), 18-26.

Fick, K.M. (1992). The influence of an animal on social interactions of nursing home residents in a group setting. The American Journal of Occupational Therapy, 47(6), 529-534.

Haddad, S., Roberge, D., \& Pineault, R. (1997). Comprendre la qualité: En reconnaître la complexité. Ruptures, revues transdisciplinaire en santé, 4(1), 59-78.

Hawley, K. (1998). Paws for comfort. Nursing, 57.

Institut national du cancer du Canada. (2000). Statistiques canadiennes sur le cancer. Toronto: Auteur.

Jonas, C., \& Feline, A. (1981). L'animal de compagnie pour le malade mental. Annales Médico-Psychologiques, 139(7), 741-747.

Jorgenson, J. (1997). Therapeutic use of companion animals in health care. Journal of Nursing Scholarship, 2(3), 249-254.

Kogan, L.R., Granger, B.P., Fitchett, J.A., Helmer, K.A., \& Young, K.J. (1999). The human-animal team approach for children with emotional disorders: Two case studies. Child and Youth Care Forum, 28, 105-121.

Landry, M., Bouchard, F., Belles-Iles, M., Gagnon, J., Gauthier, D., \& Caron, R. (2000). Programme de zoothérapie en oncologie pédiatrique: La magie d'un rêve. Centre hospitalier universitaire de Québec (document non publié).

Laurin, J. (1988). Évaluation de la qualité des soins infirmiers (2e éd.). St-Hyacinthe, QC: Edisem.

Lazarus, R.S., \& Folkman, S. (1984). Stress, appraisal and coping. New York: Springer Publishing Company.
Le devis de recherche utilisé pour cette seconde phase est un protocole expérimental à cas unique qui repose sur la comparaison du sujet avec lui-même. Ce protocole de recherche a été élaboré en collaboration avec la Faculté des sciences infirmières et l'École de psychologie de l'Université Laval. L'investigation a débuté en janvier 2003 et se fait par le biais de différentes mesures prises auprès des enfants et leur(s) parent(s), de même qu'auprès des infirmières. Les chercheures s'attendent à trouver une amélioration significative de certains symptômes chez les enfants, ainsi qu'une meilleure adaptation chez leurs parents. Les résultats de l'étude serviront à fournir d'autres données préliminaires pour la troisième phase du projet qui pourrait, à ce moment-là, impliquer au moins deux milieux de soins spécialisés en oncologie pédiatrique.

Ludwig-Beymer, P., Ryan, C.J., Johnson, N.J., Hennessy, K.A., Gattuso, M.C., Epsom, R., \& Czurylo, K.T. (1993). Using patient perceptions to improve quality care. Journal of Nursing Quality, 7, 42-51.

Mallon, G.P. (1994a). Cow as co-therapist: Utilization of farm animals as therapeutic aides with children in residential treatment. Child and Adolescent Social Work Journal, 11(6), 455-475.

Mallon, G.P. (1994b). Some of our best therapists are dogs. Child \& Youth Care Forum, 23(2), 89-101.

Martin, S. (1993). What criteria should be used for pet therapy in critical care? Critical Care Nurse, 13(2), 74.

Meisenheimer, C. (1991). The consumer: Silent or intimate player in the quality revolution. Holistic Nursing Practice, 5(3), 39-50.

Morin, D. (1997). Génériques et spécifiques: Réflexion critique sur les indicateurs de résultats en soins infirmiers. L'Appui, octobre, 4-7.

Morin, D. (1998). La qualité des soins : élaboration des indicateurs de résultats. Formation thématique, Congrès annuel OIIQ, Montréal.

Perelle, I.B., \& Granville, D.A. (1998). Assessment of the effectiveness of a pet facilitated therapy program in a nursing home setting. Extrait le 26 août 1998, de http://arrs.envirolink.org/psyeta/sa/ sa1.1/perelle.html

Poleshuck, L.R. (1997). Animal-assisted therapy for children and adolescents with disabilities. Work, 9, 285-293.

Redefer, L.A., \& Goodman, J.F. (1989). Brief report : Pet-facilitated therapy with autistic children. Journal of Autism and Developmental Disorders, 19(3), 461-467.

Ruckert, J. (1994). L'animal thérapeute (pp. 231-235). Paris: Éditions du Roseau.

Santé Canada. (1996). Mon combat pour la vie. Le cancer chez les enfants et les adolescents au Canada. Ottawa, ON: Auteur.

Saucier, A., \& Brunelle, Y. (1995). Les indicateurs et la gestion par résultats. DGPE no. 8, Collection Méthodologie et Instrumentation, Gouvernement du Québec, Ministère de la Santé et des Services sociaux, Direction générale de la planification et de l'évaluation, août 1995, 33 p.

Saylor, K. (1998). Pet visitation program. Journal of Gerontological Nursing, 24(6), 36-38.

Sheldon, L. (1997). Hospitalising children: A review of the effects. Nursing Standard, 12(1), 44-7.

St-Hilaire, C., \& Erickson, L. (1999). L'économie de la santé aujourd'hui. Comment apprécier les avantages d'une intervention de santé? La mesure de la qualité de vie. L'Actualité Médicale, (Jan.), 1.

Struck, C., \& Brady, M. (1998). Fur therapy: A home visitation program for children. Caring Magazine, May, 40-43.

Teeter, L.M. (1997). Pet therapy program : Proposal for the US department of health and human services 1996 secretary's award. Journal of American Veterinary Medical Assessment, 210(10), 1435-1438.

van Dongen-Melman, J.E., \& Sanders-Woudstra, J.A. (1986). Psychosocial aspects of childhood cancer: A review of the literature. Journal of Child Psychology and Psychiatry and Allied Disciplines, 27(2), 145-80.

Zimmer, M.J. (1980). A nursing service administrator's perspective. Nursing Research, 29(2), 98-99. 